\title{
Blood Pressure Balance as a Marker for Early Detection of Cerebral Infarction and a Criterion for Inclusion/Exclusion from Acupuncture Treatment
}

\author{
Mayumi Watanabe ${ }^{1,2^{*}}$, Yoshinobu Nakamura ${ }^{3,4^{*}}$, Chikako Tomiyama ${ }^{5}$, Nozomu Mandai ${ }^{6}$, \\ Hanaa Yousef Bakir ${ }^{7}$, Tokimasa Takeda ${ }^{1,8}$ \\ ${ }^{1}$ Faculty of Health Sciences, Kansai University of Health Sciences, Osaka, Japan \\ ${ }^{2}$ Faculty of Science and Engineering, Chuo University, Tokyo, Japan \\ ${ }^{3}$ Acupuncture Center, Nakamura Seikotsuin, Niigata, Japan \\ ${ }^{4}$ Niigata University of Health and Welfare, Niigata, Japan \\ ${ }^{5}$ Graduate School of Health Sciences, Niigata University, Niigata, Japan \\ ${ }^{6}$ Center for Humanities and Sciences, Ibaraki Prefectural University of Health Sciences, Ibaraki, Japan \\ ${ }^{7}$ Faculty of Medicine, Assiut University, Assiut Governorate, Egypt \\ ${ }^{8}$ Zinbunken, Kyoto University, Kyoto, Japan \\ Email: "watanabem62@gmail.com
}

How to cite this paper: Watanabe, M., Nakamura, Y., Tomiyama, C., Mandai, N., Bakir, H.Y. and Takeda, T. (2021) Blood Pressure Balance as a Marker for Early Detection of Cerebral Infarction and a Criterion for Inclusion/Exclusion from Acupuncture Treatment. Health, 13, 857-867. https://doi.org/10.4236/health.2021.138066

Received: July 22, 2021

Accepted: August 24, 2021

Published: August 27, 2021

Copyright $\odot 2021$ by author(s) and Scientific Research Publishing Inc. This work is licensed under the Creative Commons Attribution International License (CC BY 4.0).

http://creativecommons.org/licenses/by/4.0/

\section{(c) (i) Open Access}

\begin{abstract}
Background: Early detection of cerebral infarction (CI) by home acupuncturists might be helpful in reducing stroke mortality. We compared vital signs of patients who developed CI and those who did not to ascertain if blood pressure balance is a useful marker for the early detection of CI and for distinguishing cases with potentially effective/ineffective acupuncture treatment. Methods: In this retrospective study, vital signs such as body temperature, pulse, and blood pressure, were simultaneously obtained from the right and left arms of patients who received weekly acupuncture treatment. We focused on the differences between values obtained from the right and left arms (the balance) and compared the values of patients who developed CI [CI (+)] ( $\mathrm{n}=$ $18)$ and those of age-/gender-matched patients who did not [CI $(-)](\mathrm{n}=18)$. Results: Left/right blood pressure was significantly more unbalanced in the $\mathrm{CI}(+)$ group than in the $\mathrm{CI}(-)$ group, especially immediately before the onset of CI. Moreover, all CIs occurred on the side with a lower blood pressure. Discussion and Conclusions: Blood pressure balance might aid in the early detection of $\mathrm{CI}$ and to determine whether treatment with traditional or modern
\end{abstract}


medicine is more appropriate. Acupuncture may thus help maintain patients' quality of life.

\section{Keywords}

Acupuncture, Aged, Blood Pressure, Balance, Cerebral Infarction, Preventative Medicine

\section{Introduction}

Stroke, also known as cerebrovascular accident, is one of the major causes of death following ischemic cardiac disease [1]. In Japan, stroke is the third leading cause of death after cancer and heart disease and $86 \%$ of patients with cerebral infarction are older than 65 years [2]. Stroke is classified broadly into two categories: cerebral infarction (CI) and hemorrhage (cerebral/subarachnoid hemorrhage). CI occurs when an artery to the brain is blocked. Hemorrhages occur because of sudden rupture of arteries. In Japan, CI accounts for more than $75 \%$ of stroke [3]. Modern medicine, such as tissue plasminogen activator (tPA), has been demonstrated to be helpful in cases of acute CI when started within a few hours of CI onset [4] [5] [6]. Most patients are unaware of CI or arterial sclerosis and stroke occurs without advance warning. Therefore, early detection of CI is very difficult.

Based on modern scientific methods, recently, Sánchez-Mila et al. reported the effects of acupuncture on post-stroke recovery in a randomized controlled trial (RCT) study [7]. Many middle-aged and older people receive acupuncture therapy. Acupuncturists have the opportunity to observe patients because they regularly visit with various chronic complaints, such as pain (shoulder, back, or knees), fatigue, or rheumatoid arthritis. If home acupuncturists could promptly detect CI and closely coordinate with modern Western therapy, this would help save patient lives. Therefore, here we propose an easy and non-invasive criterion for inclusion/exclusion from acupuncture treatment and a way to detect CI while modern medicine can still help.

In this retrospective study, we examined vital signs that acupuncturists often measure (body temperature, pulse, blood pressure [BP]), which had been simultaneously obtained from the right and left arms. We focused on the difference between values from the right and left arms (the left/right balance). We compared balance between 18 patients who eventually developed CI [CI $(+)]$ and 18 age-/sex-matched patients who did not [CI $(-)]$. We found that CI $(+)$ patients presented large value in the left/right balance of BP, especially just before the CI onset. Moreover, we discovered that all CI occurred on the side in which BP was lower.

\section{Methods}

\subsection{Patients}

We conducted a retrospective study based on clinical records from patients who 
regularly attended Nakamura Seikotsuin (Niigata, Japan) between March 1997 and December 2020. We investigated the body temperature, blood pressure, and pulse rate of patients who eventually developed a CI $(n=18)$. Details of the CI (+) group are listed in Table 1 and Table 2. None was aware of their CI. At the time of analysis, these patients were receiving neither chemotherapy nor radiation therapy. They were visiting the acupuncturists to receive acupuncture related to their own complaints. Clinical records of age-matched CI (-) patients (n $=18$ ) were also examined. Written informed consent was obtained from all patients and the study was approved by the Medical Ethics Committee of Ibaraki Prefectural University of Health Sciences (Ibaraki, Japan) (e148-h30.0628).

Table 1. Patients with CI. Comparison between patients who developed CI [CI $(+)](n=$ 18 ) and those of age-/sex-matched patients who did not [CI $(-)](\mathrm{n}=18)$.

\begin{tabular}{rccccc}
\hline & CI (-) & n & CI (+) & n & p \\
\hline All & $63 \pm 13$ & 18 & $64 \pm 14$ & 18 & n.s. \\
Male & $58 \pm 12$ & 10 & $60 \pm 15$ & 10 & n.s. \\
Female & $69 \pm 11$ & 8 & $70 \pm 11$ & 8 & n.s. \\
\hline
\end{tabular}

Table 2. Details for the CI (+) group. Members of the CI (+) group opt for acupuncture treatment because of chronic disorders, such as pain in the shoulder, back, knee, or rheumatoid, or fatigue, and most are persons of middle or advanced age. All CI (+) patients had dizziness.

\begin{tabular}{|c|c|c|c|c|c|}
\hline No & Age & $\mathrm{M} / \mathrm{F}$ & Side & Chief Complaint & Dizziness \\
\hline 1 & 86 & $\mathrm{~F}$ & $\mathrm{R}$ & health care & + \\
\hline 2 & 73 & M & $\mathrm{L}$ & shoulder pain & + \\
\hline 3 & 68 & M & $\mathrm{R}$ & low back pain & + \\
\hline 4 & 31 & M & $\mathrm{L}$ & fatigue & + \\
\hline 5 & 51 & M & $\mathrm{L}$ & shoulder pain & + \\
\hline 6 & 75 & $\mathrm{~F}$ & $\mathrm{R}$ & low back pain & + \\
\hline 7 & 63 & $\mathrm{~F}$ & $\mathrm{~L}$ & shoulder/back pain & + \\
\hline 8 & 60 & M & $\mathrm{L}$ & health care & + \\
\hline 9 & 74 & $\mathrm{~F}$ & $\mathrm{~L}$ & health care & + \\
\hline 10 & 51 & $\mathrm{~F}$ & $\mathrm{R}$ & shoulder/back pain & + \\
\hline 11 & 49 & M & $\mathrm{R}$ & health care & + \\
\hline 12 & 75 & $\mathrm{~F}$ & $\mathrm{R}$ & shoulder pain & + \\
\hline 13 & 87 & M & $\mathrm{R}$ & shoulder/knee pain & + \\
\hline 14 & 59 & M & $\mathrm{R}$ & health care & + \\
\hline 15 & 58 & $\mathrm{~F}$ & $\mathrm{R}$ & health care & + \\
\hline 16 & 54 & M & $\mathrm{R}$ & health care & + \\
\hline 17 & 74 & $\mathrm{~F}$ & $\mathrm{~L}$ & neck/shoulder pain & + \\
\hline 18 & 68 & M & $\mathrm{R}$ & fatigue & + \\
\hline
\end{tabular}




\subsection{Acupuncture Application}

Stainless steel shaped as Dizhen (DZ) acupuncture needles (Soken Medical Co. Ltd., Tokyo, Japan) were used for acupuncture treatment. We consider DZ application advantageous and safe in modern clinical practice because it can avoid issues of infection and tissue damage sometimes seen with modern acupuncture needles. An autoclaved DZ needle (diameter $5.0 \mathrm{~mm}, 37.3 \mathrm{~g} / \mathrm{mm}^{2}$ ) was softly pressed vertically onto the body surface for 5 minutes at approximately 120 points from the head to the feet on both sides of the spine as reported previously [8] [9] [10] [11]. The acupuncture points corresponded to the Conception Vessel, Governor Vessel, and the Bladder Meridian including GV20 (Baihui), CV17 (Danzhong), CV12 (Zhongwan), GB20 (Fengchi), BL10 (Tianzhu), BL22 (Sanjiaoshu), BL23 (Shenshu), BL25 (Dachangshu), adding ST36 (Zusanli) and SP6 (Sanyinjiao). Electro-acupuncture stimulation (1 Hz for $20 \mathrm{~min}$, Touma NC-707, Kimura Medical Instrument, Tokyo, Japan) was followed using disposable acupuncture needles ( $40 \mathrm{~mm}$ in length, $0.2 \mathrm{~mm}$ in diameter, Seirin, Shizuoka, Japan) were inserted at the PC4 (Ximen) to modulate the autonomic nervous system (ANS) function [12]. Those points as defined by the World Health Organization [13]. Treatment was applied by nationally licensed experienced Japanese acupuncturists. The acupuncture and experiments were conducted at a constant temperature of $25.0^{\circ} \mathrm{C} \pm 0.5^{\circ} \mathrm{C}$ at a humidity of $50.0 \% \pm 5.0 \%$.

\subsection{Measurement of Body Temperature, Blood Pressure, and Pulse}

Body temperature was simultaneously measured from the right and left axilla for three minutes with clinical mercury thermometers (FAVOR, Nihon Keiryoki Kogyo Co. Ltd., Chiyoda, Japan). Blood pressure (BP) and pulse rate were also measured from the right and left arms simultaneously using two accurate automatic BP monitors with arm cuffs (NAKAMURA-BP Type II, A and D Co., Ltd, Saitama, Japan) (Figure 1(a)).

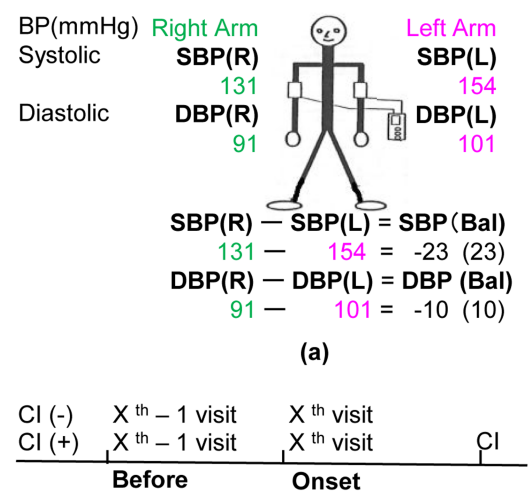

(b)

Figure 1. The method to calculate BP Balance with representative examples (a). BP Balance was defined as the difference between BP measurements from the right $(\mathrm{R})$ and left (L) arms. Two groups CI (-)/CI (+) and two-time points Before/Onset were measured to compare. As for the CI (-) group, successive two visits were observed (b). 
Measurements were conducted before the acupuncture treatment to avoid any influence of treatment. We compared these measurements between the CI ( - ) and CI (+) groups at two-time points: the last visit just before the CI onset (Onset) and a visit a couple of weeks before Onset (Before). As for the CI (-) group, successive two visits were observed (Figure 1(b)).

\subsection{BP Balance (Measuring the Difference between Right and Left Blood Pressure)}

We simultaneously assessed BP from the right (R) and left (L) arms, and calculated the $\mathrm{BP}$ balance $(\mathrm{Bal})$ as right $\mathrm{BP}$ minus left $\mathrm{BP}$, a metric that has hardly ever been used. We calculated separate balances for systolic and diastolic BP (SBP Bal and DBP Bal, respectively). The method for calculating SBP (Bal) and DBP (Bal), along with representative examples, are illustrated in Figure 1(a). In Figure 1 (a), numbers shown in parentheses are the absolute values. In short, SDP (Bal) or DBP (Bal) was positive when SBP (R) or DBP (R) was larger than SBP (L) or DBP (L), respectively. Otherwise, SDP (Bal) or DBP (Bal) would be negative. Results were shown in Figures 2-4. Figure 3 was made using absolute values and Figure 4 was made using the actual differences.

\subsection{Statistical Analysis}

Differences between values were determined by Bartlett's test, Student's t test, Welch's t test, Mann-Whitney U test, or Wilcoxon signed-rank test as appropriate. The level of significance was set at $\mathrm{p}<0.05$ and $\mathrm{p}<0.01$, and all data are presented as mean \pm SEM. All statistical tests were performed using statistical software (Statcel 2; OMS Publishing, Saitama, Japan), which is written in Visual Basic for Applications.

\section{Results}

Systolic blood pressure and diastolic blood pressure SBP (R), SBP (L), and DBP (R) tended to be higher in the CI (+) group than in the CI (-) group; however, no significant differences were found, likely because of individual variation (Figures 2(a)-(c)). In contrast, DBP (L) in the CI (+) group differed such that CI (+)-Onset was higher than both CI (-)-Before and CI (-)-Onset [CI (-)-Before vs CI (+)-Onset, $81 \pm 17 \mathrm{mmHg}$ vs $95 \pm 19 \mathrm{mmHg}, \mathrm{p}<0.05$ ] [CI (-)-Onset vs CI (+)-Onset, $80 \pm 13 \mathrm{mmHg}$ vs $95 \pm 19 \mathrm{mmHg}, \mathrm{p}<0.05$ ] (Figure $2(d))$.

\subsection{Body Temperature and Pulse}

Body temperature (axilla) tended to be lower in the CI $(+)$ group than in the CI (-) group. However, no significant difference was found because of individual differences (Figure 2(e), Figure 2(f)). In contrast, CI (-)-Onset pulse rate was significantly lower than CI (-)-Before pulse rate $(69 \pm 8$ beats/min to $65 \pm 7$ beats/min, $\mathrm{p}<0.05)$. No such difference was found in the CI $(+)$ group (Figure $2(\mathrm{~g}))$. 


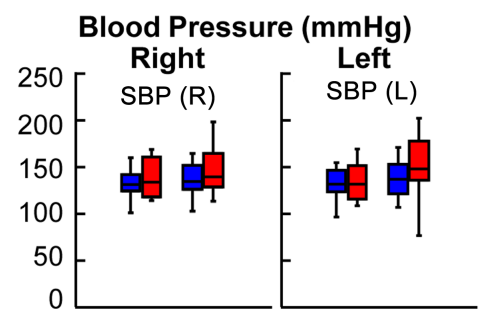

(a)

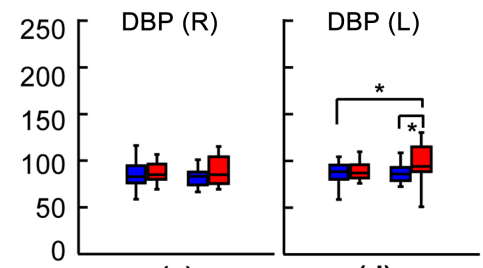

(c)

(d)

Body Temperature $\left({ }^{\circ} \mathrm{C}\right)$

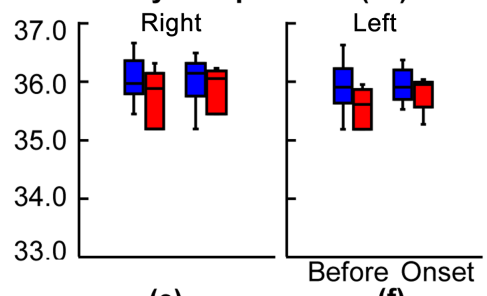

(e)

(f)

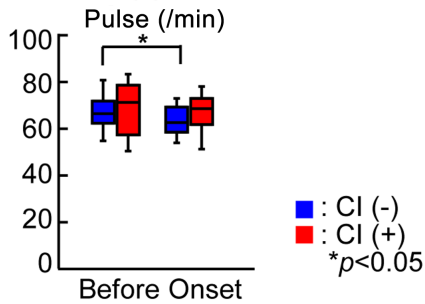

(g)

Figure 2. Blood pressure: SBP (a) and (b) and DBP (c) and (d) for the right (a), (c) and left (b), (d) arms are show for the CI (-) (blue) and CI (+) (red) groups. SBP (R), SBP (L), and DBP (R) tended to be higher in the CI (+) group than in the CI (-) group; however, no significant difference was observed ((a)-(c)). DBP (L) was higher for the CI (+)-Onset time point than for the CI $(-)$-Before or CI $(-)$-Onset time points $(\mathrm{p}<0.05$; D), Data are mean \pm SEM. SBP, systolic blood pressure; DBP, diastolic blood pressure; R, right; L, left; $\mathrm{CI}$, cerebral infarction. Body temperature: Body temperature (axilla) did not differ between the CI (+) and CI (-) groups (e), (f). Data are mean \pm SEM. Pulse rate: Pulse rate at CI $(-)$-Onset was prominently lower than that at CI $(-)$-Before $(\mathrm{p}<0.05)(\mathrm{g})$. No similar difference was seen in the CI (+) group. Data are mean \pm SEM. (a) SBP (R); (b) SBP (L); (c) DBP (R); (d) DBP (L); (e) Right; (f) Left; (g) Pulse.

\subsection{BP Balance (Absolute Value)}

The absolute BP balance was significantly higher for the CI (+)-Onset time point than the others for both SBP and DBP.

For example in SBP: (CI (-)-Before vs CI (+)-Onset, $8 \pm 7 \mathrm{mmHg}$ vs $18 \pm 22$ $\mathrm{mmHg}, \mathrm{p}<0.01$ ), (CI (-)-Onset vs CI (+)-Onset, $7 \pm 6 \mathrm{mmHg}$ vs $18 \pm 22$ $\mathrm{mmHg}, \mathrm{p}<0.01)$ and $(\mathrm{CI}(+)$-Before vs $\mathrm{CI}(+)$-Onset, $9 \pm 9 \mathrm{mmHg}$ vs $18 \pm 22$ mmHg, $\mathrm{p}<0.05$ ) (Figure 3(a)). 


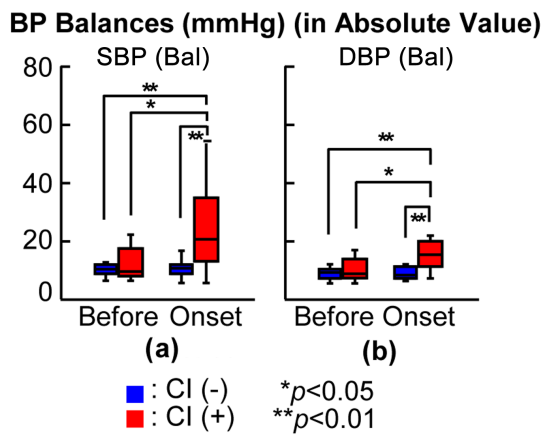

Figure 3. BP balance (absolute value): The absolute value of the BP balance at the CI (+)-Onset was significantly higher than those of the other groups/times for both SBP (Bal) (a) and DBP (Bal) (b). Data are mean \pm SEM. ${ }^{\star} \mathrm{p}<0.05,{ }^{\star *} \mathrm{p}<0.01$.

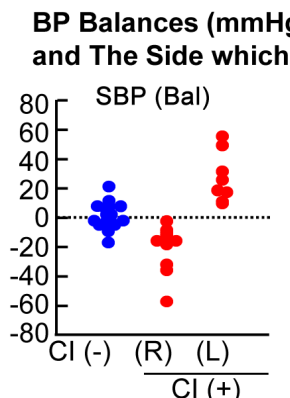

(a)

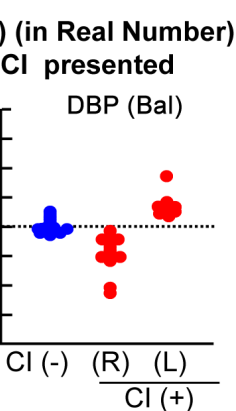

(b)

$\mathrm{Cl}(-)$ : patient without $\mathrm{Cl}$

$\mathrm{Cl}(\mathrm{R})$ : patient with $\mathrm{Cl}$ on the right side

Cl (L): patient with $\mathrm{Cl}$ on the left side

Figure 4. BP balance (actual difference and the side on which CI presented). Graphs plot the SBP (Bal) (a) and DBP (Bal) (b) balance values for those in the CI (-) group (patient without $\mathrm{CI}$ ) (blue), those with $\mathrm{CI}(\mathrm{R})$ (CI on the right side) (red), and those with $\mathrm{CI}$ (L) (CI on the left side) (red). Negative values indicate lower BP on the right. Positive values indicate lower BP on the left. All patients with $\mathrm{CI}(\mathrm{R})$ had negative balance values (low BP on the right), while those with $\mathrm{CI}(\mathrm{L})$ all had positive values (lower $\mathrm{BP}$ on the left).

On the other hand in DBP: (CI (-)-Before vs CI (+)-Onset, $5 \pm 4 \mathrm{mmHg}$ vs 15 $\pm 12 \mathrm{mmHg}, \mathrm{p}<0.01$ ), (CI (-)-Onset vs CI (+)-Onset, $4 \pm 3 \mathrm{mmHg}$ vs $15 \pm 12$ $\mathrm{mmHg}, \mathrm{p}<0.01)$ and $(\mathrm{CI}(+)$-Before vs $\mathrm{CI}(+)$-Onset, $6 \pm 5 \mathrm{mmHg}$ vs $15 \pm 12$ mmHg, $\mathrm{p}<0.05$ ) (Figure 3(b)).

\subsection{BP Balance (Actual Difference)}

In addition to absolute BP balance, we also analyzed the actual difference values (Figure 3; positive values indicate lower left BP, negative values indicate lower right $\mathrm{BP}$ ). Interestingly, $\mathrm{BP}$ balance was negative in all cases involving $\mathrm{CI}$ on the right side of the brain and was positive in all cases involving CI on the left side of the brain. In other words, we found that all CI occurred on the side with lower BP (Figure 4(a) and Figure 4(b)).

\section{Discussion}

In this study, we proposed BP balance as a new marker that may be useful for 
early detection of cerebral infarction as well as differential diagnosis for inclusion/exclusion for acupuncture treatment. Many patients routinely opt for acupuncture treatment because of chronic disorders such as pain (shoulder, back, knee, or rheumatoid), dizziness, or fatigue, and most of them are people of middle or advanced age (Table 1, Table 2). Compared with younger patients, older patients who are unaware of CI carry a high risk of CI. Therefore, acupuncturists need to be careful [14] [15].

A home acupuncturist has ongoing patient records that not only include information for traditional diagnosis, but also include modern vital signs such as body temperature, pulse rate, or blood pressure. The latter ones are regulated by the autonomic nervous system (ANS).

Dysfunction of the ANS may contribute to worse patient outcomes [16]. On the other hand, acupuncture induces somato-autonomic reflexes. In other words, acupuncture modifies unbalanced ANS function to help maintain biological homeostasis [12] [17] [18] [19]. Thus, the ANS controls the whole body to maintain internal stability and normally, both sides of the body have the same body temperature, pulse rate, and blood pressure.

We collected and compared these data on the right and the left side of the body. Because CI often presents on a single side, we investigated whether the right and left sides differ due to deficient ANS function. However, acupuncture is ineffective when ANS dysfunction is severe and irreversible [8]. In short, we need to recognize the limitations of acupuncture and criteria to inclusion/exclusion CI for acupuncture is necessary.

We propose 1) simultaneous measurement of body temperature, pulse rate, and blood pressure and 2) a way to obtain BP balance and use it to distinguish cases for which acupuncture will be effective/ineffective. This is an easy and non-invasive method that a home acupuncturist can conduct daily, and our results indicate that it might be effective.

First, the results of the BP balance analysis were as follows. The absolute value of the BP balance was significantly higher in the CI (+) group than in the CI (-) group ( $\mathrm{p}<0.05)$. CI $(+)$-Onset was much higher that $\mathrm{CI}(+)$-Before, indicating that acupuncture application was ineffective (Figure 3(a), Figure 3(b)). Thus, for people in the CI (-) group with high BP balance values, sympathetic nerves might dominate due to transient stress. In such cases, we may consider that the imbalance is at a reversible stage and there are no arterial problems. Therefore, acupuncture can decrease the BP balance value via ANS modulation.

In this study, we did not find any relationship between the degree of BP Balance and the period before CI occurred. However, all CI $(+)$ patients experienced dizziness to some degree (Table 2). The patient who had the largest CI (+) (SBP/DBP; 68/38 mmHg) had complained of shoulder pain and dizziness. Although he was unaware of a CI or arterial sclerosis, a home acupuncturist strongly advised him to visit a professional medical hospital. However, the following day he suddenly suffered a CI and died at home without any professional 
medical check. Therefore, we propose that acupuncture application may be ineffective in treating patients with highly unbalanced BP who have dizziness. In such cases, the home acupuncturist should recommend other effective treatment such as tPA and a specialized hospital as soon as possible.

Second, our results revealed that all CI presented on the same side that exhibited lower BP (Figure 4(a), Figure 4(b)). BP imbalance in the CI (+) group may thus reflect poor circulation on the lower BP side and normal circulation on the opposite side. Atherosclerotic intracranial arterial stenosis, cardiogenic embolus, narrowing of the carotid artery, or narrowing of arteries that branch from the carotid artery often present on a single side. They are thought to cause impaired blood flow and lower BP on the affected side, which leads to a high value absolute BP balance value. In this way, a patient with BP imbalance might have a severe circulatory defect and cannot expect to improve with acupuncture treatment. In this way, the BP balance metric could be a useful criterion for inclusion/exclusion from acupuncture therapy and high values could be a pre-CI warning signal.

This study has limitations. The sample size was small with large individual variation. For further study, larger sampled prospective studies that trace patients suspected of developing $\mathrm{CI}$ are needed. Additionally, BP balance values of patients who eventual develop lacunar CI should also be studied because their infarcts are small ( $2-20 \mathrm{~mm}$ in diameter) and they are caused by small vessel disease or branch atheromatous disease [20]. Moreover, we can use measures of $\mathrm{BP}$ balance to assess the effects of acupuncture or tPA treatment on post-stroke recovery.

If BP Balance is useful for distinguishing potentially effective/ineffective cases of acupuncture, we can improve early detection and treatment of CI, providing a seamless transition from acupuncture and traditional medicine to modern medicine. Patients need to know that acupuncture is not necessarily a panacea for everyone or every disease, and that a home acupuncturist has to clinically conduct a differential diagnosis to determine whether acupuncture is likely to be effective or not. Then, and only then, can acupuncture therapy help maintain patient quality of life.

\section{Conclusions}

They are thought to cause impaired blood flow and lower BP on the affected side, which leads to a high-value absolute BP balance value. Therefore, we found and continued studying BP for more than 23 years to prevent CI which most patients are unaware of.

If $\mathrm{BP}$ balance is useful for early detection of CI, we may also use it to determine whether acupuncture treatment will likely be effective or not. Moreover, blood pressure medicines cannot help a single side artery. Therefore, BP balance may provide a seamless transition from traditional to modern medicine to maintain patient quality of life. 


\section{Acknowledgements}

We thank all volunteers who participated in this study. We also thank Adam Phillips, Ph.D., from Edanz Group (http://www.edanzediting.com/ac) for editing a draft of this manuscript.

\section{Contributors}

MW and YN equally conceived the study, collected data, conducted the statistical analyses, and prepared the draft. CT contributed to the literature review of modern medicine and HB and TT contributed to the review of traditional medicine. NM contributed to the statistical analysis. All authors critically edited drafts of this manuscript and approved the final manuscript.

\section{Ethics Approval}

This study was approved by the Medical Ethics Committee of Ibaraki Prefectural University of Health Sciences (Ibaraki, Japan) (e148-h300628).

\section{Funding}

This study was supported by The Uchida Energy Science Promotion Foundation (Grant Number 3-1-085) and the Japan Society for the Promotion of Science's Grants-in-Aid for Scientific Research (Kakenhi Grant No. 19K10727).

\section{Conflicts of Interest}

The authors have no competing interests to report.

\section{References}

[1] World Health Organization (2018) The Top 10 Causes of Death, Global Health Observatory (GHO) Data. World Health Organization, Geneva. http://www.who.int/news-room/fact-sheets/detail/the-top-10-causes-of-death

[2] Japanese Ministry of Health, Labour and Welfare. Annual Health, Labour and Welfare Report H29 (for the Year of 2016). https://www.mhlw.go.jp/english/wp/wp-hw11/dl/summary.pdf

[3] Yamaguchi, S. and Kobayashi, S. (2014) Epidemiology of Acute Stroke in Japan: Japan Standard Stroke Registry Study. Japanese Journal of Stroke, 36, 378-384. https://doi.org/10.3995/jstroke.36.378

[4] Ford, G.A., Ahmed, N., Azevedo, E., Grond, M., Larrue, V., Lindsberg, P.J., et al. (2010) Intravenous Alteplase for Stroke in Those Older than 80 Years Old. Stroke, 41, 2568-2574. https://doi.org/10.1161/STROKEAHA.110.581884

[5] Mateen, F.J., Buchan, A.M. and Hill, M.D. (2010) Outcomes of Thrombolysis for Acute Ischemic Stroke in Octogenarians versus Nonagenarians. Stroke, 41, 1833-1835. https://doi.org/10.1161/STROKEAHA.110.586438

[6] Galldiks, N., Zaro-Weber, O., Dohmen, C., Neveling, M., Haupt, W.F., Fink, G.R., et al. (2010) Systemic Thrombolysis with rt-PA in Patients under 40 Years of Age: A Subgroup Analysis of the Cologne Stroke Experience. Cerebrovascular Diseases, 30, 514-518. https://doi.org/10.1159/000319776

[7] Sánchez-Mila, Z., Salom-Moreno, J. and Fernández-de-las-Peñas, C. (2018) Effects 
of Dry Needling on Post-Stroke Spasticity, Motor Function and Stability Limits: A Randomised Clinical Trial. Acupuncture in Medicine, 36, 358-366. https://doi.org/10.1136/acupmed-2017-011568

[8] Watanabe, M., Takano, O., Tomiyama, C., Matsumoto, H., Urahigashi, N., Kainuma, E., et al. (2012) The Effects of Application of an Ancient Type of Acupuncture Needle on Body Temperature, Immune Function and the Autonomic Nerve System. Health, 4, 775-780. https://doi.org/10.4236/health.2012.410120

[9] Watanabe, M., Takano, O., Tomiyama, C., Guan, J., Hou, G., Mori, H., et al. (2013) The Effects of Application of an Ancient Type of Acupuncture Needle on Increase in Urination of Hospitalized Oldest-Old People. Health, 5, 1092-1098. https://doi.org/10.4236/health.2013.57147

[10] Watanabe, M., Nakamura, Y., Tomiyama, C. and Abo, T. (2016) A Specific Pattern in the Basal Body Temperature Chart during the First Week of Pregnancy May Warn of a Miscarriage Crisis. Health, 8, 723-729. https://doi.org/10.4236/health.2016.88075

[11] Watanabe, M., Nakamura, Y., Tomiyama, C., Fu, H., Shimaya, M., Ling, Y., et al. (2016) The Association between Self-Monitored Reproductive Parameters, Pregnancy Outcomes and Acupuncture Treatment. Health, 8, 730-736.

[12] Nishijo, K., Mori, H., Yosikawa, K. and Yazawa, K. (1997) Decreased Heart Rate by Acupuncture Stimulation in Humans via Facilitation of Cardiac Vagal Activity and Suppression of Cardiac Sympathetic Nerve. Neuroscience Letters, 227, 165-168.

[13] Huang, L.X. (2010) Standard Acupuncture Point Locations: WHO Standard Acupuncture Point Locations in the Western Pacific Reason. People's Medical Publishing House, Beijing, 23-224.

[14] Ishiga, S. and Watanabe, K. (2011) The Effectiveness of Acupuncture and Moxibustion Therapy Based on the Primordial Oriental Medicine in a Patient with Numbness after Cerebral Infarction. Journal of Mind-Body Science, 20, 17-31.

[15] Fujii, R. and Yano, T. (2013) The Rate of Patients Undergoing Acupuncture and Moxibustion-Comparison between the Rate of Treatment with Acupuncture and Moxibustion and That of Treatment with Acupuncture, Moxibustion, and Anma-Massage-Shiatsu. The Bulletin of Meiji University of Integrative Medicine, 8, 1-12.

[16] Divani, A.A., Liu, X., Di Napoli, M., Lattanzi, S., Ziai, W., James, M.L., Jafarli, A., Jafari, M., Saver, J.L., Hemphill, J.C., Vespa, P.M., Mayer, S.A. and Petersen, A. (2019) Blood Pressure Variability Predicts Poor In-Hospital Outcome in Spontaneous Intracerebral Hemorrhage. Stroke, 50, 2023-2029.

[17] Mori, H., Nishijo, K., Kawamura, H. and Abo, T. (2002) Unique Immunomodulation by Electro-Acupuncture in Humans Possibly via Stimulation of the Autonomic Nervous System. Neuroscience Letters, 320, 21-24. https://doi.org/10.1016/S0304-3940(02)00012-5

[18] Takahashi, N., Nakamura, T., Kanno, N., Kimura, K., Toge, Y., Lee, K.H., et al. (2011) Local Heat Application to the Leg Reduces Muscle Sympathetic Nerve Activity in Human. European Journal of Applied Physiology, 111, 2203-2211. https://doi.org/10.1007/s00421-011-1852-0

[19] Nakamura, Y., Watanabe, M., Tomiyama, C. and Oh, Z. (2020) A Medical Hypothesis: Pulse Diagnosis of Traditional Chinese Medicine Indicates the Status of Autonomic Nervous System. Chinese Traditional Medicine Journal, 3, 1-3. http://traditionalmedicinejournals.com/

[20] Wardlaw, J.M. (2005) What Causes Lacunar Stroke? Journal of Neurology, Neurosurgery and Psychiatry, 76, 617-619. 